Slavica

bruxellensia

\section{Slavica bruxellensia}

Revue polyphonique de littérature, culture et histoire

slaves

$3 \mid 2009$

Pensée des hommes

\title{
Entretien avec Alexandre Avelitchev
}

\author{
Katia Vandenborre et Eric Metz
}

URL : http://journals.openedition.org/slavica/204

DOI : $10.4000 /$ slavica.204

ISSN : 2034-6395

Éditeur

Université libre de Bruxelles - ULB

Édition imprimée

Pagination : 74-86

ISSN : 2031-7654

\section{Référence électronique}

Katia Vandenborre et Eric Metz, «Entretien avec Alexandre Avelitchev », Slavica bruxellensia [En ligne], 3 | 2009, mis en ligne le 15 juin 2009, consulté le 19 avril 2019. URL : http://journals.openedition.org/ slavica/204 ; DOI : 10.4000/slavica.204

Ce document a été généré automatiquement le 19 avril 2019

\section{(c) (i) $\odot$}

Les contenus de Slavica bruxellensia sont mis à disposition selon les termes de la Licence Creative Commons Attribution - Pas d'Utilisation Commerciale - Pas de Modification 3.0 France. 


\title{
Entretien avec Alexandre Avelitchev
}

\author{
Katia Vandenborre et Eric Metz
}

\section{Présentation}

1 Alexandre Avelitchev est né en 1946 à Almaty. Docteur ès Lettres de l'Université de Moscou, il enseigne aujourd'hui le russe à la faculté de traduction et d'interprétation de l'Université Mons-Hainaut (Belgique). Son nom est toutefois avant tout lié aux anciennes éditions Progress, qu'il a dirigées de 1982 à 1996. Il fut premier vice-président de l'Association des éditeurs de la Russie de 1990 à 1996, il est président à vie de l'Association des éditeurs universitaires russes. Cela fait de lui tant un témoin privilégié qu'un acteur de l'histoire de l'URSS'.

2 Au nom de la rédaction de «SLAVICA BRUXELLENSIA », nous le remercions chaleureusement pour l'entretien qu'il nous a accordé.

\section{Entretien}

\section{Les années d'enfance}

Katia Vandenborre : Avant tout, nous serions curieux de savoir dans quel univers vous avez grandi...

À l'époque à laquelle je suis né, Almaty², qui est une ville située à trois cents kilomètres de la Chine, était un vivier d'ethnies et de cultures. Des théâtres, des studios de cinéma de Moscou ou de Leningrad y avaient été déplacés pendant la guerre. Pour ne donner qu'un exemple, le célèbre film Ivan le Terrible de Serguej Ejzenštejn a été tourné là-bas. Des gens y avaient déménagé pendant la guerre, d'autres y avaient été contraints. Des Tchétchènes avaient été expulsés vers le Kazakhstan et l'Asie Centrale en 1944 : selon une logique purement surréaliste, on appelait leur quartier «China Town ». Il y avait aussi des Chinois puisque la frontière était proche, mais également des Allemands de la Volga qui avaient été déplacés là-bas, des Ouighours, une minorité opprimée en Chine, ou encore des milliers de Coréens expulsés d'Extrême-Orient. Tout ceci faisait un 
cocktail de nations qui apportait un cachet tout à fait exceptionnel. Je pense que tous ceux qui ont vécu dans cette région à la même époque que moi sont atteints du virus très résistant de l'internationalisme naturel, non idéologique.

C'est à Almaty aussi que je suis allé à l'école, dont je garde de très bons souvenirs. Cela n'avait rien à voir avec la morne image de l'école communiste type. Nous avions un théâtre, un club des Amateurs d'art, un cercle poétique où nous lisions, entre autres, Anna Ahmatova, Osip Mandel'štam et tant d'autres...

Eric Metz : Vous avez grandi fin des années 1950 et dans les années 1960, donc pendant la déstalinisation. Que retenez-vous de cette époque?

Mes souvenirs les plus éloignés existent sous forme de sensations, d'images vivantes ou de photos couleur. Les plus anciens remontent à l'époque où j'avais 4 ou 5 ans. Les conditions de vie étaient difficiles, mais on ne s'en rend compte qu'avec le recul. L'enfance ne connaît pas de nuages. Sitôt après ma naissance, en 1946, mon père est parti vivre avec une autre femme. Nous avons vécu avec ma mère, ma grand-mère et mon beau-père dans une baraque aménagée dans d'anciennes écuries. En fait, chaque appartement occupait la place de deux ou trois box de chevaux qui avaient été nettoyés et retapés pour les besoins de locataires humains. Il n'y avait pas d'eau à l'intérieur, les commodités étaient dehors. Malgré ce décor plus que modeste, je me souviens non pas de la pauvreté, mais surtout d'une grande solidarité humaine. Au moment des fêtes ou des périodes de deuil, les gens sortaient de la nourriture et des bouteilles et s'installaient dehors autour d'une longue table qui était aussi longue que notre baraque. C'était une époque de multiples tragédies humaines (mon grand-père maternel, comme des millions d'autres, a disparu dans une prison stalinienne, à Tachkent, en 1939), de drames de toutes sortes, mais tout le monde restait soudé. Ces souvenirs à répétition ont marqué mon esprit à tout jamais. À la mort de Josif Stalin, tout le monde pleurait, ceci est connu. Moi aussi, je m'en souviens très bien de ce moment. Ce jour-là, il faisait gris et, derrière les nuages, il y avait d'incessants bruits d'avions. Tout le monde pensait que c'était probablement la guerre. À la fin des années 1950, je l'ai senti : mes parents ont commencé à respirer.

\section{Études à Moscou}

\section{K. V. : Quand êtes-vous arrivé à Moscou?}

Après l'école à Almaty, j'ai voulu poursuivre mon cursus à Moscou: j'ai décidé de devenir philologue. Ma mère ne voulait pas que je parte, elle souhaitait me voir faire mes études à la faculté d'Almaty. Mais moi, je voulais partir. Je n'avais pas encore 17 ans quand je suis parti « conquérir Moscou ». J’y ai fait mes études, à la faculté de Lettres de l'Université Lomonosov. J'avais le français et l'espagnol comme langues étrangères. Mais, en plus, comme j'étais passionné par les langues, j'ai suivi, pendant deux ans, les cours de spécialisation en linguistique comparative. J'ai donc fait du sanscrit, du grec, du latin, du lituanien: cela faisait partie de la formation solide qu'on recevait à l'époque.

E. M. : Vous avez donc fait vos études pendant les dernières années du « dégel » politique et culturel de Nikita Hruščëv...

Je vous ai parlé un peu de cette tournure d'esprit que j'avais grâce à mon enfance passée à Almaty... D'une certaine manière, l'éloignement de la capitale stimule la lecture. Au moment de venir à Moscou, j'avais déjà lu tout ce qui a été publié à ce 
moment par Aleksandr Solženicyn, Vasilij Aksënov, ILja Erenburg, Jurij Dombrovskij (vivant, d'ailleurs, à cette époque, à Almaty). La revue littéraire «PROSTOR » (aussi d'Almaty) m'a fait découvrir les œuvres interdites d'Andrej Platonov... J'étais curieux, intéressé par des tas de choses, et Moscou offrait beaucoup à ceux qui y vivaient, à l'époque. Quand, en octobre 1964, Hruščëv a dû quitter ses fonctions, je l'ai ressenti comme une offense personnelle. C'est naïf, mais c'était comme je vous le raconte. Je me souviens d'une manifestation à deux, que nous avons organisée avec un copain. Nous sommes allés vers l'ambassade de Chine qui se trouve dans le quartier de l'Université en criant : « Honte à vous les Chinois, rendez-nous Hruščëv ! ». Le policier de garde rigolait en nous observant. Nos amis nous demandaient: «Mais pourquoi devant l'ambassade de Chine?». Pour nous c'était clair: les Chinois n'avaient jamais pardonné la déstalinisation. Mao Tsé-Tung n'a jamais voulu entretenir des rapports avec Hruščëv.

\section{E. M. : Est-ce la seule manifestation à laquelle vous avez participé ?}

J'ai toujours été assez actif. Par honnêteté. Pas du tout par excès de courage. Je me

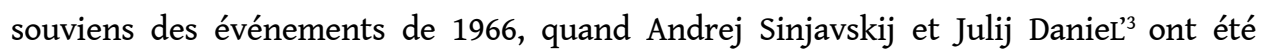
arrêtés. Un groupe d'étudiants, dont je faisais partie, avait organisé une action à la Faculté, ayant pour but d'exprimer nos griefs aux autorités. Pour le faire, nous avons demandé un entretien avec le doyen, nous sommes venus à cette rencontre avec une pétition signée par la majorité des étudiants. Nous exigions que le professeur Duvakin, spécialiste de Majakovskij, ne soit pas puni : par décision du Conseil de la Faculté, il lui avait été interdit de continuer à donner ses cours car il avait témoigné au procès de Sinjavskij en faveur de ce dernier. Quand, au procès, on lui avait demandé ce qu'il pensait d'Andrej Sinjavskij, il l'avait expliqué en ces termes : « Après la fin de la guerre je donnais mes cours et, je me souviens, un jour, un soldat vêtu de sa tunique militaire est entré dans la salle. Il était modeste, il m'a demandé s'il pouvait rester. Je lui ai donné mon accord. Il est resté, et il est revenu à chaque cours. Et, avec le temps, devant mes yeux, ce petit vilain petit canard est devenu un beau cygne ». Rien que pour ça, le professeur Duvakin a été licencié. C'est donc pour défendre celui-ci que nous avions organisé cette action. Mais le contexte général laissait deviner le reste. Mon mobile dans cette affaire était purement humain, et puis seulement politique. Vu que le professeur Duvakin donnait les cours à la faculté et vu que sa fille était dans mon groupe, je me sentais personnellement touché par cette affaire. Ne pas intervenir était inadmissible...

\section{E. M. : Il y a eu des suites?}

Nous avons vu de nos propres yeux quelques officiers du KGB rendre visite au doyen. Grande fut notre surprise de voir que, pour nous, il n'y eut pas de suite. Je suis parti travailler en Algérie l'année suivante, en 1967. Jusqu'à la dernière minute, je n'y croyais pas puisque j'avais signé mon nom en toutes lettres...

\section{E. M. : Vous avez donc eu l'occasion de voyager hors de l'URSS ?}

J'ai énormément voyagé dans ma vie. Mais l'Algérie, c'était la destination de mon premier voyage à l'étranger. Ce pays était officiellement considéré, à l'époque, comme un État ami. D'ailleurs, je l'aime toujours. Le président Houari Boumediene promettait de construire le socialisme arabe. Tout le monde faisait semblant d'y croire. La coopération technique s'élargissait et les étudiants du département français (garçons de préférence) y étaient envoyés pour travailler en tant que traducteurs/interprètes. 
K. V.: Dans quelle direction se sont développés vos intérêts littéraires et scientifiques pendant vos études?

Je ne sortais pratiquement pas de la bibliothèque de littérature internationale, où je lisais une quantité inimaginable de livres et d'articles. C'est ainsi que j'ai découvert (assez tôt) la revue "TEL QUEL ${ }^{4}$, dont j'ai lu tous les numéros parus. J'en ai ressorti l'idée qu'il était possible d'analyser et d'interpréter les textes autrement (je n'avais pas encore lu à ce moment-là les écrits d'OPOJAZ ${ }^{5}$ ). Cette petite découverte m'a beaucoup servi plus tard. À part cela, j'ai traduit quelques ballades de François Villon, des sonnets de Pierre de Ronsard ainsi que « La cantatrice chauve » d'Eugène Ionesco pour la mettre en scène à l'Université. J'ai toujours adoré tout ce qui était absurde : l'absurdité cachée du monde devient évidente quand elle est mise à nu par une parole absurde. Pour moi c'est quelque chose de très important. J'ai ensuite écrit une thèse sur la métaphore dans un contexte extra-poétique. Je l'ai défendue en 1973. J'en suis assez fier car j'ai réussi à proposer une approche d'analyse rhétorique qui est encore d'actualité.

\section{Progress}

\section{E. M. : Comment êtes-vous devenu éditeur?}

Après ma thèse, j'ai commencé à travailler car j'avais une famille à nourrir. J'ai donné des cours de littérature à Orehovo-Zuevo ${ }^{6}$. C'était assez loin de Moscou, donc ce que je gagnais là-bas, je le dépensais pour payer mes billets de train. Cela m'occupait. J'ai fait cela pendant une année. Ensuite, j'ai commencé à travailler à l'Université de Moscou.

La carrière d'éditeur m'a trouvé par hasard : le recteur de l'Université, l'académicien Rem Hohlov, que je connaissais à peine, voulait absolument que je vienne remplacer l'ancien directeur des éditions universitaires qui approchait l'âge de la retraite. Le premier vice-recteur Jevgenij M. Sergeev m'aurait entendu intervenir dans une conférence et avait décidé que j'étais un candidat idéal pour le poste de directeur. Après avoir obtenu des informations précises sur moi, le rectorat m'a proposé d'accepter l'offre. Toutefois, je ne connaissais pas le métier et me sentais nullement attiré par lui. Finalement un compromis fut trouvé : notre gentlemen's agreement avec Hohlov était très peu réaliste et présumait que je travaillerais aux éditions cinq ans maximum après quoi on me laisserait revenir à mon métier de professeur. La réalité fut tout autre: je suis resté dans l'édition plus de 20 ans, ayant découvert un incroyable continent de création et cette excitante complexité intellectuelle et psychologique qui caractérisent le monde de l'édition. À la mort de Leonid Brežnev, le Comité Central du Parti, par une décision signée par un des nouveaux membres du Politburo, un certain Mihaïl Gorbačëv, m'a transféré au poste de directeur littéraire des éditions Progress, la plus grande maison d'édition de l'URSS.

\section{K. V. : Pouviez-vous, dans votre travail d'éditeur, prendre la température du régime?}

Oui et non. Il faut préciser les termes. Pour un éditeur soviétique, un des indicateurs importants pour sentir le souffle des changements en gestation a toujours été la politique de la censure. Pendant les années que j'ai passées dans l'édition, j'ai pu constater plusieurs fois un mouvement de balancier : un affaiblissement progressif du contrôle suivi de son brusque renforcement et vice versa. Mais soyons clairs: les maisons d'édition pour lesquelles j'ai travaillé étaient très différentes. Les éditions de l'Université étaient très académiques et bénéficiaient d'une autonomie que les autres ne possédaient (peut-être) pas. Au début des années 1980, la censure ne s'intéressait 
plus aux ouvrages de physique, de mathématique, de philologie etc. Le profil de Progress et sa place dans le paysage de l'édition soviétique étaient bien différents. Le contrôle de la part de la censure existait pour elles aussi, mais il était beaucoup moins contraignant, moins agressif, jamais répressif. Je trouve, au moins, deux explications à ce phénomène. En travaillant avec des partenaires occidentaux, nous ne pouvions pas rester assis sur nos dogmes. À l'époque, c'était d'ailleur un de mes arguments de travail. De plus, les responsables de Progress côtoyaient les décideurs lors de réunions au Comité Central ou ailleurs. Les règles du jeu étaient différentes. Les liens personnels facilitaient parfois la publication de certains ouvrages qui, autrement, n'auraient jamais vu le jour.

\section{E. M. : Quelle était la mission de Progress comparativement aux autres maisons d'édition?}

Fondées en 1931, les éditions Progress étaient bien connues à l'étranger en tant que maison-héritière de la Camaraderie d'édition des ouvriers étrangers en URSS. La maison gardait des traditions remontant à l'époque du Komintern (1919-1943). Plusieurs leaders communistes de l'avant-guerre (Maurice Thorez, Dolorès Ibarruri, Georgij Dimitrov et tant d'autres) y avaient travaillé. La publication en langues étrangères des classiques du marxisme et, plus tard, d'ouvrages politiques modernes s'était élargie au cours des années par la programmation (en langues étrangères) de manuels universitaires, de dictionnaires, de belles lettres, d'albums illustrés, etc. Tout ceci ne représentant qu'une seule facette de la pyramide, celle tournée vers l'extérieur du pays.

Des traductions vers le russe de multiples ouvrages d'auteurs étrangers constituait un autre axe de production. Grâce à cette activité de grande qualité, bon nombre d'idées novatrices et de noms mondialement connus (parmi lesquels Ilya Prigogine, Arnold Toynbee, Roman Jakobson, Mircea Eliade, et plus tard Georges Orwell, Aldous Huxley, Alexis de Tocqueville, Sigmund Freud, Karl Jung pour ne citer qu'eux) avaient été mis en circulation intellectuelle et scientifique en URSS. Pour cet effort, Progress a toujours été apprécié par l'intelligentsia et était à la limite considéré comme une maison à laquelle on "autorise des choses». Cette apparente facilité n'existait cependant que dans l'imagination des observateurs extérieurs.

Enfin, le troisième volet des activités de la maison consistait à publier, en langue russe, des ouvrages dits « secrets » ou, pour être plus clair, « antisoviétiques ».

K. V. : À partir de quel moment?

Je suis arrivé en 1982 et cela existait déjà depuis une quinzaine d'années. De ces ouvrages il n'en sortait parfois qu'un seul exemplaire. Ainsi, un jour, j'ai reçu un coup de fil du Comité Central, on m'a demandé de lire un livre en italien, d'en faire un résumé et de rester discret : c'était pour l'usage personnel de Konstantin Černenko. Ce livre le tournait en ridicule et, comme je le trouvais ridicule, moi aussi, je ne l'ai pas épargné...

\section{E. M. : Avez-vous eu connaissance des réactions du lecteur?}

Non, je n'ai pas eu cet honneur. De manière générale, tous ces ouvrages nous étaient commandés par le Comité Central, le KGB, le Ministère de la Défense ou le Ministère des Affaires étrangères et étaient analysés au sein de leurs cellules. Cela était destiné à de très hauts échelons du pouvoir. 


\section{K. V. : Y avait-il parfois des fuites de ces livres?}

Naturellement. Pourtant tous les exemplaires étaient numérotés et envoyés par courrier spécial. Et malgré cela, il y a eu des fuites, déjà au début des années 1980.

\section{K. V. : Comment connaissiez-vous ces livres antisoviétiques ?}

C'était très simple. Quand je travaillais aux éditions Progress, j'avais un budget assez confortable pour acheter des livres. Habituellement, nous passions des contrats avec les épouses des ambassadeurs qui, souvent, n'étaient pas débordées de travail... Nous les chargions d'acheter des livres dans les librairies et de les envoyer par la valise diplomatique. Parfois, nous recevions un livre quelques jours à peine après sa sortie à New York, à Londres ou à Paris. Comment nous les connaissions? Par les catalogues. Par ailleurs, Progress participait régulièrement à toutes les grandes Foires du livre à travers le monde.

\section{E. M. : En ce qui concerne les livres étrangers publiés en russe, qui faisait la sélection?}

Comme chaque grande maison d'édition, nous avions des comités de lecture, des gens qui nous renseignaient sur tel ou tel livre... Il y avait en tout quatorze rédactions, dont chacune avait son comité de lecture. Moi, je dirigeais, entre autres, la collection linguistique. Nous avons publié des volumes de Jakobson, de Nikolaj Trubeckoj, une nouvelle traduction d'Edward Sapir dont la première édition des années 1930 était absolument introuvable.

\section{K. V. : Quel était votre rôle en tant que directeur de Progress ?}

Il est difficile de répondre en quelques mots. Le rôle de l'éditeur en URSS n'était pas le même que celui de l'éditeur en Occident. Rien d'étonnant, car le pays lui-même était bien différent des autres. Donc nous faisions beaucoup de choses que l'on ne faisait pas ici. Très souvent, par exemple, je servais de parasol ou de paratonnerre pour protéger les têtes de mes collègues... Et c'était important pour les collaborateurs. Quand il y avait un problème, au Comité Central, avec la publication d'un livre, j'étais là pour calmer le jeu. Je n'ai jamais puni mes subordonnés. De manière globale, je définissais la politique éditoriale, en créant moi-même un certain nombre de collections, en trouvant des auteurs, etc. Mes fonctions ont évolué avec le temps et les besoins. Les années 1988-1993 ont constitué une période cruciale dans la transformation et le renouvellement de Progress, et en même temps elles sont devenues son âge d'or. Avec la tombée des interdictions de la censure, il fallait bien lancer une discussion sur les sujets antérieurement tabous : les malaises économiques majeurs de l'URSS de la fin des années 1980, des données sociologiques inédites, des analyses impitoyables sur l'état des sciences sociales étouffées par les dogmes, de violentes polémiques autour de la théorie marxiste et les perspectives du socialisme en URSS figuraient parmi les sujets les plus chauds. Les textes d'auteurs étaient publiés dans leur intégralité, sans coupures, ni censure. De cette époque datent la biographie de Nikolaj Buharin par l'historien américain Stephen Cohen, la biographie de Stalin par Robert Tucker, le livre Bolsheviks come to power de Alexander Rabinowitch de l'Indiana University, l'Histoire de la Russie soviétique de Nicolas Werth et des dizaines d'autres ouvrages qui documentaient le passé soviétique et qui provoquèrent tous des débats dans la presse et dans la société.

\section{K. V. : Progress avait donc un certain pouvoir d'initiative?}

Je dirais même, un pouvoir certain. Il y a plein de livres qui ont été créés sur l'initiative de Progress. C'était, par exemple, le cas de la collection « Dialogue Est-Ouest », qui a été très difficile à faire démarrer car personne ne voulait en assumer la responsabilité dans 
le cas d'éventuelles difficultés. Je me suis occupé personnellement de cette collection, du choix des auteurs. Finalement, nous avons réussi à publier une dizaine de titres, à commencer par un dialogue avec le sociologue suisse Jean Ziegler. Pour le convaincre, je l'ai assuré que pas une seule ligne n'allait être modifiée sans son accord. J'ai tenu ma parole. Ce qui ne fut pas le cas de Jean. Parallèlement à l'édition intégrale en russe, un éditeur suisse publie le livre à Lausanne et me l'envoie. Que ne lis-je pas dans la présentation du livre: « Dans la présente édition, des passages qui ont été censurés en URSS ont été rétablis en italique... ». Je ne comprenais pas, j'avais tout fait pour que rien ne soit modifié. Plus encore, j'apprends que Ziegler a fait la même blague avec les éditions allemande et italienne que j'avais contactées pour lui. Aujourd'hui, ça n'a plus d'importance. Mais à l'époque, je l'ai mal vécu et j'ai publié une réplique assez mordante dans la "LiTERATURNAJA GAZETA ». Puis, nous avons travaillé avec John Kenneth Galbraith, le célèbre auteur de la théorie de la convergence, selon laquelle le socialisme et le communisme allaient un jour se rencontrer et fusionner. Sa théorie était difficilement défendable en URSS. Le dialogue a eu beaucoup de succès. Il y a eu aussi Yasuhiro Nakasone, le premier ministre japonais. Mais c'est déjà une autre histoire...

\section{E. M. : Quel a été le sort réservé à ces livres publiés en tirage très restreint?}

Pour être bref: ils ont passé au domaine public et sont devenus accessibles à tout le monde. En 1988, j'ai envoyé une lettre au Comité Central demandant l'autorisation de remettre tous ces livres portant des mentions « confidentiel ", « top secret ", etc., à la Bibliothèque Nationale. Avant d'écrire, j'ai demandé l'avis et le soutien à Georgij Šahnazarov, l'assistant de Gorbačëv. Il a parlé à ce dernier, qui a donné son accord de principe. Nous avons aussi republié un certain nombre de titres plus tard. Le 1984 de Orwell a été publié pour la première fois, à un tirage limité, en 1984. En 1988, ce roman a été tiré à 200000 exemplaires.

E. M. : Est-ce que d'autres titres remarquables, que vous avez publiés au début des années 1980, ont connu de gros tirages par la suite?

Nous l'avons fait, par exemple, avec la traduction de l'histoire de la révolution russe en quatorze volumes de Edward Carr. Voici une petite anecdote, si vous le permettez. Un jour, en 1990, je reçois un coup de fil du Kremlin, de la part de Aleksandr Nikolaevič Jakovlev, membre du Politburo, qui me demande si je peux passer chez lui. On s'installe tous les deux, avec nos tasses de thé, à une petite table près de la porte d'entrée (« Là, ils ont des oreilles ", me dit-il, en baissant la voix et en indiquant la direction de son grand bureau). Après quoi, il me demande : «Alexandre, combien te faut-il de temps pour publier les quatorze volumes de Carr?». Moi: «Est-ce qu'il y aura le financement?» Lui : «Il te faudrait combien?» Moi : «Je ne saurais pas vous dire comme ça. Mais ça coûtera assez cher... Quel tirage voulez-vous?». Lui : " Mille cinq cents, mais il faut faire très vite. " Je réfléchis : «Quatorze volumes, il nous faudra une quarantaine de traducteurs - historiens...» Lui: «Tu peux le faire en douze mois? ».Moi : «Ca devrait pouvoir se faire.». Nous l'avons fait en six mois car j'avais compris que si on ne le faisait pas maintenant, on ne le ferait peut-être jamais. Nous ne savions pas de quoi l'avenir allait être fait. Un peu plus tard, après la chute de l'URSS, les deux premiers volumes ont été publiés à grand tirage. Puis, c'est devenu tellement cher qu'il n'y a plus eu moyen de publier les autres. 
K. V. : Étant donné la place du livre dans la société russe, pensez-vous avoir joué un rôle catalyseur dans la perestroïka?

Progress, sûrement. D'ailleurs Gorbačëv l'a écrit dans ses Avant-mémoires. À propos, savez-vous le nom dont ma maison a été honorée par la presse internationale à la fin des années 1980 ? La « Maison Glasnost ». J'en suis fier, car je sais que j'y suis pour quelque chose.

K. V.: Sur la Bibliothèque virtuelle «Znamja », la rédactrice Natalja Ivanova vous surnomme «le destructeur de Progress ». Qu'en pensez-vous?

Je pense que je pourrais vous renseigner encore une dizaine de sites russes qui reprennent, depuis déjà plus de dix ans, les mêmes mots, les mêmes phrases et les mêmes accusations, à quelques virgules près. Puisque j'ai vécu et miraculeusement survécu dans l'œil de ce cyclone qui a détruit le premier Groupe d'édition indépendant de Russie (cité à l'époque comme un exemple de la privatisation honnête et réussie), je sais qu'il s'agit d'un mensonge. Mais votre question me montre que ce mensonge est toujours mieux entendu que la vérité. Je vous dois donc une brève explication. Entre 1992 et 1995, l'économie russe est passée par une phase terrible : $1440 \%$ d'inflation en 1992. Les prix augmentaient, les ventes chutaient. Pourtant, il fallait payer pour la production des livres, payer les auteurs, les salaires tous les mois. Nous avions alors huit cents employés. De plus, j'avais à financer un fond pour les vétérans de Progress, créé deux ans avant le début des réformes. On s'en sortait haut la main. Jusqu'en 1994. Cette année-là nous avons investi beaucoup plus dans la production de livres que ce que nous avons pu en récupérer. Le pays, le marché changeaient. Il fallait innover, diversifier, tout essayer pour survivre et aller de l'avant...

\section{Nouvelles voies}

E. M. : À cette époque mouvementée qu'étaient les années 1990, avez-vous essayé d'autres voies, hors de l'univers de d'édition?

J'aimais bien la vie d'éditeur et j'ai fait tout ce que j'ai pu pour que Progress réussisse. La devise du Groupe Progress était : la diversification. Après le putsch de 1991, ayant observé la paralysie des mass-médias officiels pendant les quelques jours de ces événements tragiques, la décision de créer une compagnie de télévision a été prise. Il s'agissait de TVProgrès, qui produisit des émissions politiques pour la première chaîne de télévision. Comme dans le cas des livres, le genre très populaire à l'époque était celui du dialogue avec des personnalités du monde entier : Nakasone, Lech WałĘsa, Alexandr Ruckoj, le vice-président russe opposé à Boris EL'cin, le président ukrainien Leonid Kravčuk, et tant d'autres. Cela marchait très bien au niveau de l'audimat. Par contre, cela ne rapportait pratiquement rien. Le marché des compagnies de production n'était pas encore développé en Russie. En se formant, un peu plus tard, il est vite devenu maffieux.

Après avoir créé une dizaine de nouvelles sociétés au sein du Groupe, nous avons lancé des projets de partenariat avec des grandes maisons internationales. C'est ainsi que sont nées les entreprises mixtes: Hachette-Progrès, Reader's Digest Russie, Russian Britannica, le magazine international pour les hommes d'affaires « BUSINESS IN THE USSR/ RUSSIA » avec le Groupe Hersant, la Librairie du Progrès et tant d'autres.

À un moment, nous avons eu un projet de film ${ }^{8}$ L'idée du film n'était pas tout à fait neuve. Depuis un certain nombre d'années, j'avais dans mon tiroir, un scénario écrit 
par Jean-Louis Gouraud, un très bon ami, auteur, globe-trotter, journaliste et... ami de Bartabas. Une fois le financement trouvé (Progress n'investissait pas un cent), nous avons finalisé le contrat avec un célèbre producteur français, Marine Karmitz (MK2). Le Groupe Progress devenait producteur général et MK2 coproducteur. Le réalisateur Bartabas, accompagné par l'auteur du scénario, partit en repérage en Sibérie, en Iakutie, dans la région du lac Baïkal, etc. Le calendrier du tournage fut préparé, le casting réalisé, le matériel acheté ou loué et c'est là que NovosibirskProfbank, la Banque qui avait signé le contrat de financement du film, jeta l'éponge... La directrice me demande de prendre un crédit pour maximum trois mois. Elle remboursera tout après cette échéance... et, avec les intérêts demandés par le créancier.

\section{K. V. : Ceci constituait une nouvelle tournure dans le scénario de départ?}

C'est exact. Un tournant tant imprévu que dramatique. Comment pouvait-on savoir que NovosibirskProfbank était déjà en train, en secret, de préparer le dépôt de son bilan et agissait de mèche avec la banque qui est devenue notre créancier. Nous avons remboursé les trois quarts de la dette en trois mois, comme ceci avait été prévu, tout en proposant à notre créancier, la ResursBank, de calculer les intérêts pour payer le solde le mois suivant. Mais l'argent ne les intéressait plus, c'est l'immeuble et tous les avoirs financiers du Groupe qui se trouvaient dans le collimateur. Difficile à imaginer, en dehors de la Russie. Le scénario qui a suivi était banal pour l'époque: menaces personnelles, découragement, blocage de tous les comptes bancaires, campagne de diffamation dans la presse, filature, saisie des biens, encore des menaces, promesses, tromperies, tentatives d'occuper le bâtiment par la force... C'est là que mon enfer personnel a commencé. Je n'ai pas compris à temps que le pays qui était le mien n'existait plus. Avec sa disparition se sont évaporées les valeurs morales qui servaient de base aux habitudes d'affaires. La loi ne protégeait plus contre la pression des bandits enrichis. À un certain moment, je suis allé rencontrer le Président de la Cour d'Arbitrage de la Fédération de Russie, un homme sage et triste sous le poids de ses connaissances de la vie. Il était au courant de notre affaire et m'a dit, après m'avoir écouté : «Vous savez, la loi est de votre côté, mais vous allez perdre l'affaire. » Nous avons effectivement tout perdu malgré tous les efforts et toutes les évidences.

Des tournants, il y en eu d'autres. L'Etat restait jusqu'au dernier moment un actionnaire du Groupe. Mais l'État a tout fait pour m'écarter de l'affaire, pour priver Progress de toute aide nécessaire. À la dernière Assemblée générale des actionnaires du Groupe, en novembre 1996, après plusieurs mois de pression médiatique, les représentants de l'État ont appelé les actionnaires à voter contre moi. J'ai obtenu environ $65 \%$ des votes. Je le dis pour vous montrer que l'avis exprimé par NataLja Ivanova (une bonne critique littéraire, d'ailleurs) n'est pas partagé par tout le monde qui connait cette affaire.

\section{K. V. : Et c'est à ce moment-là que vous êtes parti en Belgique...}

Non, beaucoup plus tard. Je suis têtu et je ne voulais pas quitter le champ de bataille en vaincu. J'avais encore des illusions. On n'envisageait pas de partir, ni mon épouse, ni moi. Après tous ces cataclysmes, j'ai cherché à me reconstruire pendant trois ans. J'ai travaillé par ci, par là, organisé, pour le compte de la mairie de Moscou, un Festival d'arts et métiers pour les écoliers. Ceci ne m'intéressait pas vraiment. Mais il fallait choisir : ou bien cultiver en moi un complexe, ou bien oublier ce que j'avais été et repartir à zéro. J'ai choisi la deuxième solution. Avec ma femme Dominique nous sommes partis en Belgique, en 1999. Tout était à construire. Je crois que nous avons réussi cet examen. 


\section{K. V. : Comment en êtes-vous arrivé à travailler à l'Université de Mons ?}

Dans ma vie, le hasard a joué un grand rôle. Parfois j'ai l'impression d'avoir vécu plusieurs vies. Comme ma femme est Montoise, nous avons ici notre port d'attache. Ma femme, historienne de l'art, travaillait aussi pour plusieurs éditeurs, à notre retour elle a été engagée par les éditions La Renaissance du livre. Moi, je cherchais du travail, je me suis inscrit à des formations. A un certain moment, j'ai réalisé des études de marchés du livre en Pologne, au Maroc, en Tunisie, etc. pour le groupe De Boeck et l'AWEX ${ }^{9}$. Puis, un jour, on m'a proposé une place à l'université. J'ai immédiatement accepté. J'ai rasé ma barbe blanche et je me suis ce poste à l'université. Pour y arriver, il a fallu quand même accomplir un parcours du combattant : je n'ai jamais obtenu la reconnaissance de mon diplôme de doctorat. J'ai donc suivi des cours d'aptitude pédagogique (CAPAES) à l'ULB pendant un an. C'était une perte de temps, mais cette "spécialisation » m'a été vitale pour ma carrière académique.

E. M. : J'aurais une dernière question en guise de conclusion. Auriez-vous voulu et pu être directeur d'une maison d'édition en Russie aujourd'hui ?

Non. La Russie actuelle n'est plus le pays que j'ai connu. Chaque fois que j'y retourne, je me sens perdu en ressentant cette distance qui m'en sépare aujourd'hui. La dernière fois que j'y suis allé, c'était en novembre 2008, j'ai senti à quel point je ne suis plus de là-bas. Et je ne suis pas d'ici non plus. Avec l'âge, ma femme et moi nous nous sentons de plus en plus devenir de véritables citoyens du monde. Il y a beaucoup d'endroits sur cette Terre, où nous sommes chez nous. Nous sommes très bien à Almaty, à Samara, à Saint-Pétersbourg, à Casablanca, à Kairouan ou à Tunis, dans les petites villes de province, mais plus à Moscou. Ce qui a rendu ma vie à la fois facile et difficile, c'est le fait que je suis un rêveur de nature. Après la débâcle de Progress, il m'a fallu passer à autre chose. Je l'ai fait. Quand je rencontre aujourd'hui des éditeurs russes, à la foire de Francfort ou ailleurs, ils me disent tous qu'avant c'étaient des belles années, que ça vaudrait la peine que je revienne... Revenir, mais revenir où et pourquoi ?

\section{NOTES}

1. La rédaction accède à la demande de $M$. Avelitchev en ne retranscrivant pas son nom selon le système de translittération qu'elle utilise habituellement.

2. Almaty, anciennement Alma-Ata, est la principale ville du Kazakhstan.

3. Arrêtés en septembre 1965, le critique Andrej Sinjavskij (pseud. : Abram Terc) et le traducteur Julij DanieL' (pseud. : Nikolaj Aržak) furent accusés d'avoir envoyé des manuscrits à l'étranger. Ils furent condamnés respectivement à sept et cinq ans de réclusion.

4. «TEL QUEL » est une revue de littérature d'avant-garde fondée aux éditions du Seuil à Paris par des intellectuels tels que Jean-Edern Hallier et Philippe Sollers.

5. «Obščestvo izučenija POetičeskogo JAZyka », la Société pour l'étude de la langue poétique fut fondée en 1916 par un groupe de linguistes et de critiques littéraires parmi lesquels on peut compter Viktor Šklovskij, Boris Ejhenbaum et Osip Brik. OPOJAZ développa une théorie littéraire dite « formaliste ». 
6. Ville industrielle située à $90 \mathrm{~km}$ à l'est de Moscou.

7. Voir http://infoart.udm.ru/magazine/znamia/set/ivanova/ivano016.htm.

8. Alexandre Avelitchev a coproduit le film Chamane, réalisé par Bartabas sur la base du scénario de Jean Louis Gouraud.

9. L'Agence wallonne à l'Exportation et aux Investissements étrangers (AWEX) est le département de la Région wallonne (Belgique) en charge de la promotion du commerce extérieur et de l'accueil des investisseurs étrangers.

INDEX

Index géographique : Belgique, Mons, Russie, Moscou

Index chronologique : communisme, post-communisme

Mots-clés : Université de Mons, Progress

\section{AUTEURS}

\section{KATIA VANDENBORRE}

Doctorante à l'Université Libre de Bruxelles (Belgique) et à l'Université de Varsovie (Pologne) ; membre du comité de rédaction de « Slavica Bruxellensia »

\section{ERIC METZ}

Université de Gand (Belgique), Haute Ecole Artesis (Anvers, Belgique) ; membre du comité de rédaction de « Slavica Bruxellensia » 